\title{
A Questionnaire-Based Study to Investigate the Extent of Shared Decision-making During Consultations in Out-of-hours Primary Care
}

\author{
Samuel Finnikin, $\mathrm{MSC}^{7}$ (D) , Joanne Protheroe, $\mathrm{PhD}^{2}$, and Daniel Lasserson, $\mathrm{MD}^{1,3}$ \\ 'Institute of Applied Health Research, University of Birmingham, Birmingham, England, UK; ${ }^{2}$ School of Primary, Community and Social Care, Keele \\ University, Keele, UK; ${ }^{3}$ Dept of Acute Medicine, City Hospital, Sandwell and West Birmingham Hospitals, Birmingham, UK.
}

$\mathrm{J}$ Gen Intern Med 35(8):2513-5

DOI: $10.1007 / \mathrm{s} 11606-019-05515-4$

(c) Society of General Internal Medicine 2019

\section{INTRODUCTION}

Out-of-hours $(\mathrm{OOH})$ primary care physicians (PCPs) provide urgent primary care when in-hours practices are closed. During consultations, several decisions may be made about treatments, investigations or referral. In modern healthcare systems, there is growing emphasis on involving patients in decisions about their care. It is generally agreed that this should be achieved through the process of 'shared decisionmaking' (SDM). It is also understood that patients vary in their preferences for involvement in decision-making. ${ }^{1}$

In $\mathrm{OOH}$ care, the patient and clinician are not known to one another, there are little or no medical records and patients tend to present with acute problems. These factors mean there is no pre-existing relationship or implicit knowledge in the encounter which increases the necessity for eliciting and incorporating values and preferences into the consultation through a SDM approach. It is not known currently whether decisions being made in an $\mathrm{OOH}$ setting are being shared with patients or not. In other healthcare settings, studies suggest that the degree of patient involvement is generally low, especially in the absence of tools to promote SDM. ${ }^{2}$

This research will, for the first time in the urgent primary care setting, establish the degree to which patients want to be involved in decision-making and how much they feel involved in the decisions made about their health during consultations with $\mathrm{OOH}$ clinicians.

\section{METHODS}

This was a questionnaire-based study which established patient preferences for decision-making and the degree of shared

$\overline{\text { Prior Presentation Preliminary findings were reported in an oral presen- }}$ tation at the South West meeting of the Society for Academic Primary Care in Southampton in 2019.

Received October 1, 2019

Accepted October 21, 2019

Published online November 11, 2019 decision-making experienced by the patient using validated tools (Control Preference Scale (CPS) ${ }^{3}$ and CollaborRATE ${ }^{4}$ respectively). The questionnaire was administered to competent adult patients attending three $\mathrm{OOH}$ treatment centres across Birmingham (England), after their consultation with a PCP or Advanced Nurse Practitioner. Control Preference Scale ratings were described and summary statistics of CollaboRATE scores were calculated $(0-4$ in three domains; maximum score of 12) along with the proportion of responses that recorded a maximum CollaboRATE score (as this 'top score' reporting method is used in comparable studies ${ }^{5,6}$ ). The Kruskal-Wallis test was applied to identify if there was a significant difference between CollaboRATE scores for CPS categories.

\section{RESULTS}

There were 120 questionnaires completed from 147 patients approached (response rate $81.6 \%$ ). Respondent characteristics and CPS responses are shown in Table 1. The median CollaboRATE score was 9.5 (IQR 7.5-12). Maximum scores were returned by $46(38.3 \%, 30.1-47.3 \%)$ of respondents. Figure 1 shows the association between CPS and CollaboRATE. As patient preferences shift towards more control of the decisionmaking process, they are less satisfied with the degree of decision sharing in the consultation. The difference between categories is significant $(p=0.018)$.

Table 1 Respondent Characteristic and Decision Control Preference

\begin{tabular}{lll}
\hline \hline & & $\boldsymbol{N}(\boldsymbol{\%})$ \\
\hline Gender & Male & $38(31.7)$ \\
& Female & $82(68.3)$ \\
Age group & $18-27$ & $36(30.0)$ \\
& $28-37$ & $27(22.5)$ \\
& $38-47$ & $21(17.5)$ \\
& $48-57$ & $20(16.7)$ \\
& $58-67$ & $11(9.2)$ \\
Decision control preference & $68-77$ & $3(3.3)$ \\
& $78-87$ & $1(0.8)$ \\
& Active & $4(3.3)$ \\
& Active shared & $23(19.2)$ \\
Total & Collaborative & $48(40.0)$ \\
& Passive shared & $21(17.5)$ \\
& Passive shared & $24(20.0)$ \\
& & $120(100)$
\end{tabular}




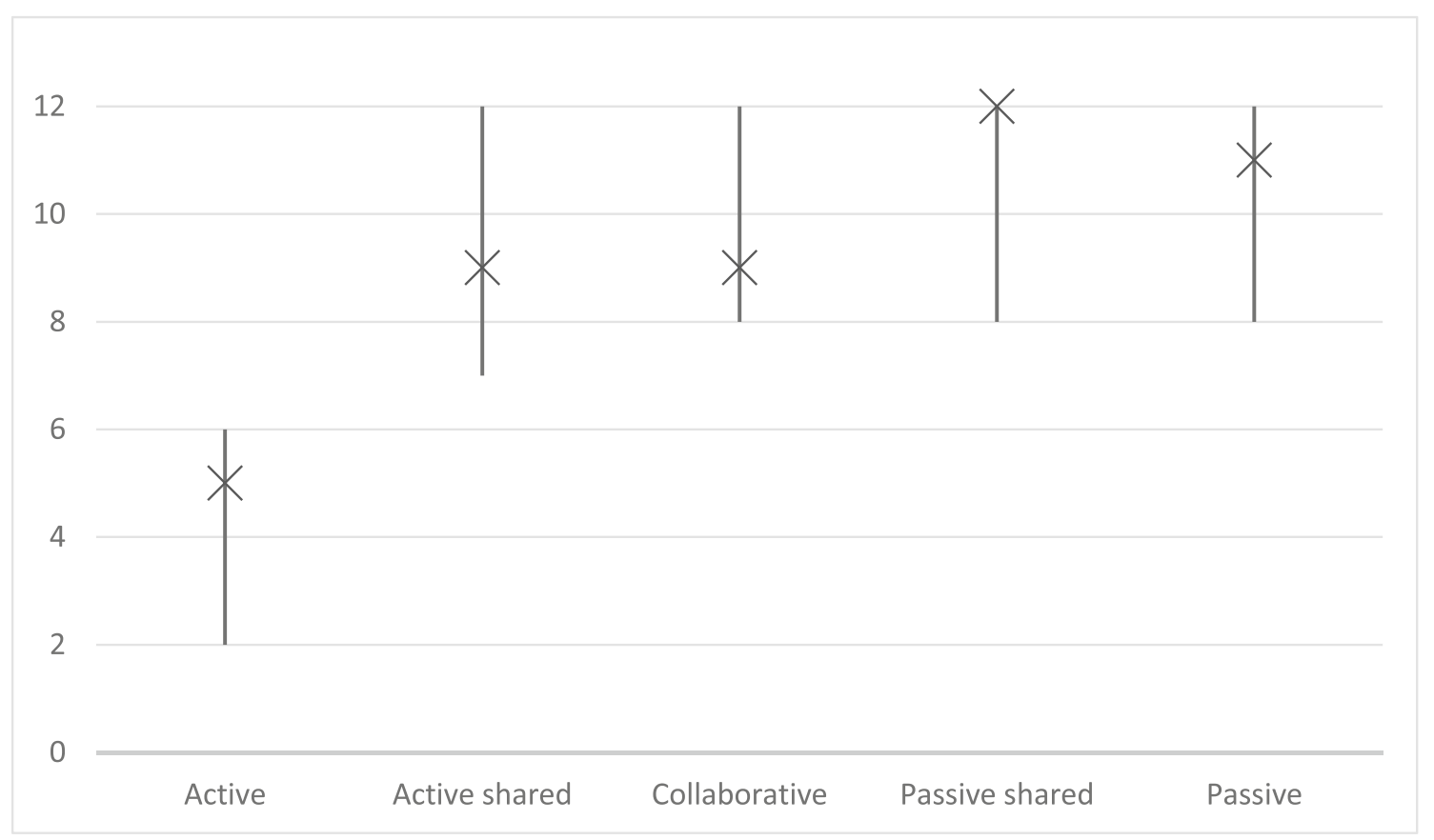

Figure 1 Median CollaboRATE scores by decision preference.

\section{DISCUSSION}

This study shows that patients are experiencing shared decision-making in $\mathrm{OOH}$ primary care. However, when compared with similar tools in other settings, the results suggest that decision sharing could be improved. ${ }^{5,6}$ Most patients expressed a preference for a collaborative approach to decision-making, and, although some preferred a more active role, a larger minority preferred a passive approach. Patients who prefer an active decision-making role reported a lower perception of decision sharing. As the patients did not get to choose the clinicians they saw, this suggests that the same consulting approach is perceived differently depending on decisionmaking preference. Those patients who wanted to participate more in the decisions may not have felt they got this opportunity. However, patients who were more passive in their approach were more satisfied with the degree of decision-making, which is understandable if the degree if sharing is the same but the appetite from the patient to get involved in decisions is reduced.

This study highlights the difficulty in measuring SDM in practice. Without understanding how patients prefer to make decisions, it is difficult to interpret metrics that are framed in one approach to decision-making, an approach that may not be congruent with individual patients' preferences. As health systems around the world focus on improving SDM, there is a need to better understand SDM measures and how to incorporate individual control preferences so we can accurately assess the impact of interventions on patient care. This will ensure that investment in SDM results in tangible and positive changes for patients.
Acknowledgements: This study would not have been possible without the support of the clinicians and directors of Badger Group.

Corresponding Author: Samuel Finnikin, MSc; Institute of Applied Health Research University of Birmingham, Birmingham, England, UK (e-mail: s.j.finnikin@bham.ac.uk).

Author Contributions SF DL and JP were involved in the design of the study. SF undertook data collection and analysis and all authors contributed and approved the final manuscript.

Funding Information This study was unfunded but we acknowledge the support of the West Midlands Clinical Research Network who funded the work required facilitate this research.

\section{Compliance with Ethical Standards:}

Ethical approval was received from the Proportionate Review Subcommittee of the London Bueen Square Research Ethics Committee (REC ref 18/LO/1425).

Conflict of Interest: $S F$ receives income from freelance writing, lecturing and reviewing from a variety of sources including EBSCO Information Services who produce Option Grid ${ }^{\mathrm{TM}}$ decision aids.

\section{REFERENCES}

1. Kiesler DJ, Auerbach SM. Optimal matches of patient preferences for information, decision-making and interpersonal behavior: evidence, models and interventions. Patient Educ Couns 2006;61(3):319-41.

2. Couët $\mathbf{N}$, Desroches $\mathbf{S}$, Robitaille $H$, Vaillancourt $H$, Leblanc A, Turcotte S, et al. Assessments of the extent to which health-care providers involve patients in decision making: a systematic review of studies using the OPTION instrument. Health Expect 2015;18(4):542-61.

3. Degner LF, Sloan JA. Decision making during serious illness: what role do patients really want to play? J Clin Epidemiol 1992;45(9):941-50. 
4. Elwyn G, Barr PJ, Grande Sw, Thompson R, Walsh T, Ozanne EM. Developing CollaboRATE: a fast and frugal patient-reported measure of shared decision making in clinical encounters. Patient Educ Couns 2013;93(1):102-7.

5. Barr PJ, Forcino RC, Thompson R, Ozanne EM, Arend R, Castaldo MG, et al. Evaluating CollaboRATE in a clinical setting: analysis of mode effects on scores, response rates and costs of data collection. BMJ Open 2017;7(3):e014681.
6. Forcino RC, Barr PJ, O’Malley AJ, Arend R, Castaldo MG, Ozanne EM, et al. Using CollaboRATE, a brief patient-reported measure of shared decision making: Results from three clinical settings in the United States. Health Expect 2018;21(1):82-9.

Publisher's Note Springer Nature remains neutral with regard to jurisdictional claims in published maps and institutional affiliations. 